\title{
A Model Membrane Platform for Reconstituting Mitochondrial Membrane Dynamics
}

Yifan Ge ${ }^{1}$, Sivakumar Boopathy ${ }^{1}$, Adam Smith ${ }^{2}$, Luke H. Chao ${ }^{1,3}$

${ }^{1}$ Department of Molecular Biology, Massachusetts General Hospital ${ }^{2}$ Department of Chemistry, University of Akron ${ }^{3}$ Department of Genetics, Harvard Medical School

\section{Corresponding Author}

Luke H. Chao

chao@molbio.mgh.harvard.edu

\section{Citation}

Ge, Y., Boopathy, S., Smith, A.,

Chao, L.H. A Model Membrane Platform

for Reconstituting Mitochondrial

Membrane Dynamics. J. Vis. Exp. (163), e61620, doi:10.3791/61620 (2020).

\section{Date Published}

September 2, 2020

DOI

$10.3791 / 61620$

URL

jove.com/video/61620

\section{Abstract}

Mitochondrial dynamics is essential for the organelle's diverse functions and cellular responses. The crowded, spatially complex, mitochondrial membrane is a challenging environment to distinguish regulatory factors. Experimental control of protein and lipid components can help answer specific questions of regulation. Yet, quantitative manipulation of these factors is challenging in cellular assays. To investigate the molecular mechanism of mitochondria inner-membrane fusion, we introduced an in vitro reconstitution platform that mimics the lipid environment of the mitochondrial inner-membrane. Here we describe detailed steps for preparing lipid bilayers and reconstituting mitochondrial membrane proteins. The platform allowed analysis of intermediates in mitochondrial inner-membrane fusion, and the kinetics for individual transitions, in a quantitative manner. This protocol describes the fabrication of bilayers with asymmetric lipid composition and describes general considerations for reconstituting transmembrane proteins into a cushioned bilayer. The method may be applied to study other membrane systems.

\section{Introduction}

Membrane compartmentalization is a hallmark of eukaryotic cells $^{1}$ (Figure 1A). Biological membranes are increasingly recognized as more than a two-dimensional solvent, and are considered as an environment playing critical roles in regulating protein function and macromolecular complex assembly ${ }^{2,3}$. Native lipids are ligands that regulate membrane protein activity ${ }^{3,4}$. Membrane spatial organization and the ability of membranes to be sculpted into diverse shapes are important physical properties for selecting new functions ${ }^{3,5}$.

Model membrane platforms are biomimetic systems that can help us understand cellular membrane structure, dynamics, and function $6,7,8$. Model membranes typically comprise a lipid mixture of well-defined composition, with defined biophysical properties (stiffness, thickness, and elasticity). Coupled to fluorescence imaging, model membrane platforms allow quantitative analysis of membrane structure and 
function $9,10,11$. Lipid bilayer reconstitution strategies have been used to study SNARE-mediated membrane fusion ${ }^{9}, 10$ DNA-mediated membrane fusion ${ }^{12}$, and viral fusion ${ }^{11,13}$. An advantage of such methods is the potential to obtain kinetic information for intermediate steps preceding an observable reaction event ${ }^{14}$.

The plasma membrane has been extensively studied using model membranes. Bilayers with lipid phase separation have been developed to study lipid raft structures important in cellular signaling 11,15,16. Micropatterned lipid planar bilayers $^{17}, 18$ have been used to investigate the organization of cell receptors. Polymer or gel-supported membranes have been used as biomimetic systems to study the membranecytoskeleton organization, membrane protein partitioning during cell signaling, and migration at cell-cell contacts ${ }^{19}$.

Artificial membrane systems are also being applied to study subcellular organelles ${ }^{20}$. Organelles feature characteristic morphologies that create distinct sub-environments. The endoplasmic reticulum (ER) network is one example. Upon reconstitution of reticulons into liposomes, tubular membrane structures with properties similar to the cellular ER are formed $^{21}$. The addition of atlastin, an ER fusion protein, can induce lipid tubules from liposomes to form a network ${ }^{20}$. This is one example for how proteoliposomes can provide functional insight into organelle morphology and dynamics.

Mitochondrial membrane fusion and fission are essential for the health of the mitochondrial population $22,23,24,25$.
A set of dynamin family GTPases catalyzes mitochondria membrane fusion. Mfn 1/2 catalyzes outer-membrane fusion. Opa1 mediates inner-membrane fusion ${ }^{26}$ (Figure 1B). Opa1 has two forms: a long form (I-Opa1), transmembraneanchored to the mitochondrial inner-membrane, and a 'soluble' short form (s-Opa1), present in the intermembrane space. The ratio of the two Opa1 forms is regulated by the

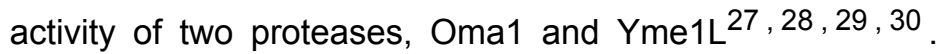
Important questions in Opa1 regulation include: how the two forms of Opa1, (short and long) mediate membrane fusion and their regulatory interplay $28,29,31,32,33$.

Here we describe a reconstitution strategy successfully applied to investigate mitochondrial inner-membrane fusion that clarified the roles of I- and s-Opa1 in inner-membrane fusion. We developed a platform mimicking the mitochondrial inner-membrane using a polymer-tethered lipid bilayer and $200 \mathrm{~nm}$ unilamellar vesicles. The benefits of a polymer tether beneath the lipid bilayer include the following. First, it preserves the reconstituted transmembrane protein, which would otherwise may be disrupted by the proximity to the glass slide ${ }^{34}$. Secondly, it serves a thick water layer between the lipid bilayer and glass substrate, which facilitates studies of pore opening ${ }^{9}$, and thirdly the viscoelastic nature of the PEG polymer allows membrane curvature changes ${ }^{35}$. We used three-color fluorescence imaging to characterize steps in membrane fusion (Figure 1C-F). 

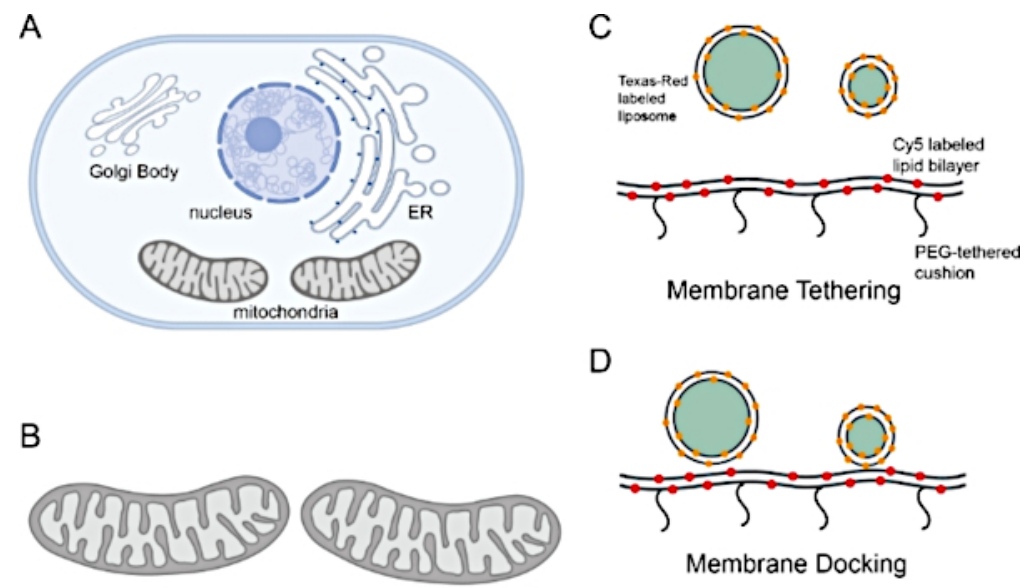

$\mathrm{D}$
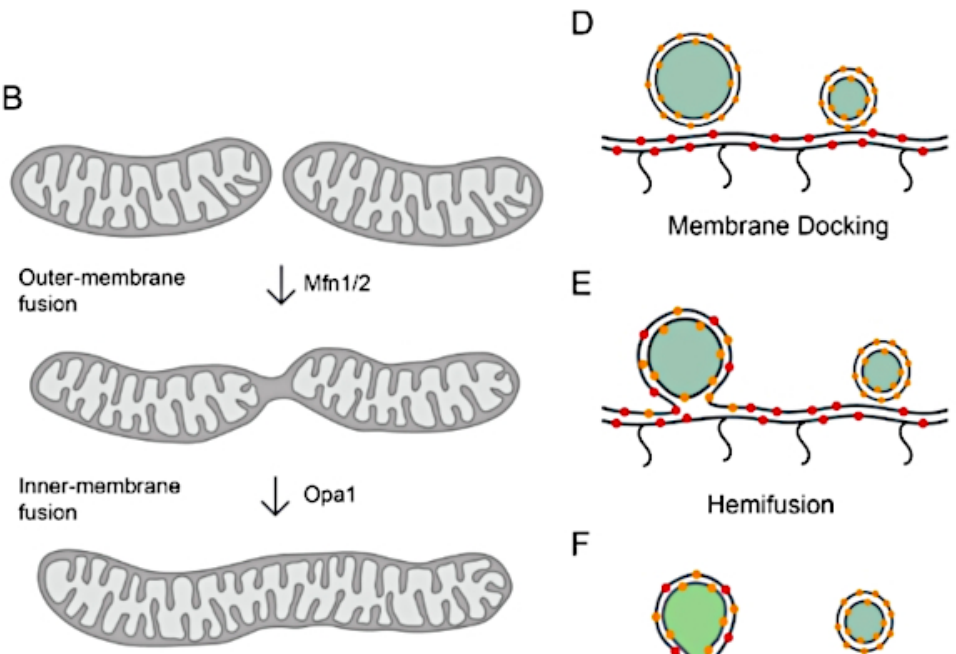

E

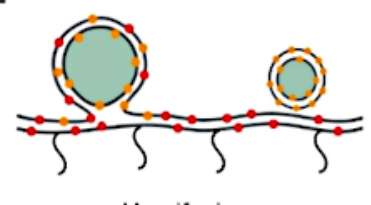

$\mathrm{F}$

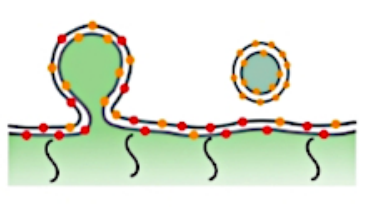

Content Release

Figure 1: Monitoring mitochondrial membrane fusion.

(A) Organelles are cellular membrane compartments. (B) Sequential steps of mitochondrial membrane fusion. Fusion of the outer membrane of mitochondria is catalyzed by Mfn1 and/or Mfn2, while inner-membrane fusion is mediated by Opa1. (C-F) Schematic of the in vitro reconstitution platform to study mitochondrial membrane fusion. The platform includes two parts: a proteoliposome and a polymer-tethered lipid bilayer, both with reconstituted I-Opa1. Fluorescent labels, including two different fluorescent membrane dyes and a content marker, help distinguish steps during membrane fusion. The two membrane markers (Cy5-PE (red) and TexasRed PE (orange) make a FRET pair, which can report on close membrane docking. Diffusion of TexasRed-PE that labels proteoliposome is an indicator of lipid demixing (hemifusion). Content release is monitored through the dequenching of the calcein signal (shown in green). Panels $A$ and $B$ created using Biorender.

Please click here to view a larger version of this figure. 


\section{Protocol}

\section{Preparation of lipid mixtures}

1. Prepare a lipid stock solution by dissolving 1,2-dioleoylsn-glycero-3phosphocholine (DOPC), 1-palmitoyl-2oleoyl-sn-glycero-3-phosphoethanolamine (POPE), La-phosphatidylinositol (Liver PI), cardiolipin, and 1,2-dioleoyl-sn-glycero-3-phosphoethanolamine-N[methoxy(polyethylene glycol)-2000] (18:1 PEG2000 PE) into chloroform at the concentration of $25 \mathrm{mg} / \mathrm{mL}$. Dissolve fluorescent dye-conjugated lipid (TexasRed DHPE and Cy5 DOPE) chloroform at a concentration of $1 \mathrm{mg} / \mathrm{mL}$. Store the lipid solution in amber vials with chloroform resistant liner, further sealed with polytetrafluoroethylene tape. The solution can be kept at $-20{ }^{\circ} \mathrm{C}$ for up to 6 months.

2. Make solutions $A$ and $B$.

1. Mix lipid to prepare solution A (final concentration 1 $\mathrm{mg} / \mathrm{mL})$ that contains DOPC (52.8 mol\%), POPE (20 mol\%), Liver PI (7 mol\%) and cardiolipin (20 mol\%), and $0.2 \mathrm{~mol} \%$ fluorophore.

2. Make solution B (final concentration $1 \mathrm{mg} / \mathrm{mL}$ ) containing DOPC (47.8 mol\%), POPE (20 mol\%), liver PI (7 mol\%), cardiolipin (20 mol\%) and DOPEPEG2000 (5 mol\%), and $0.2 \mathrm{~mol} \%$ fluorophore.

3. Generate the lipid mixture by adding the calculated volume of storage solution into amber vials using a glass syringe. Match the final volume by adding extra chloroform into the vials.

NOTE: For FCS (fluorescence correlation spectroscopy), decrease the ratio of dye conjugated lipid to $0.002 \mathrm{~mol} \%$ and replace the rest by DOPC.

\section{Fabrication of lipid bilayers}

1. Bake microscope cover glass slides at $520{ }^{\circ} \mathrm{C}$ for 30 min. After baking, cool down the cover slides to room temperature.

2. Weigh approximately $10 \mathrm{~g}$ of sodium hydroxide and add to $500 \mathrm{~mL}$ of methanol while stirring. Stir for $2 \mathrm{~h}$, continue to add sodium hydroxide in the solution until precipitates start to show. Make sure to wear appropriate PPE during the whole process.

3. Clean the glass slides in $10 \%$ sodium dodecyl sulfate solution; methanol saturated with sodium hydroxide; and $50 \mathrm{mM}$ hydrochloric acid, sequentially (bath sonication under each condition for $30 \mathrm{~min}$ ). Clean the glass slide in ultrapure water for $10 \mathrm{~min}$ between each condition.

NOTE: Though highly recommended to use fresh solutions for glass slide preparation, each solution can be reused up to $5 x$ or within 1 month, whichever comes first. Make sure to stir-mix the solution before each use.

4. Store the cleaned cover glass sealed in $\mathrm{HCl}$ solution up to 2 weeks to ensure good bilayer quality. If stored in ultrapure water, use the slides within a week.

5. Clean the polytetrafluoroethylene trough of the LangmuirBlodgett dipping system using chloroform and ultrapure water until no wetting is observed on the trough. Spray chloroform on the trough surface, wipe thoroughly with cellulose wipes $3 x$. Rinse with ultrapure water and remove the water via suctioning. Repeat $3 x$.

6. Cover the surface of the trough with clean ultrapure water.

7. Take 2 pieces of surface-treated cover glass from cleaning solution or ultrapure water, and rinse the glass slide with ultrapure water for approximated $30 \mathrm{~s}$. 
8. Place the cover glass in a back-to-back manner. Use the substrate clamp to hold the glass slides. Immerse the glass slide underneath the water surface by manually clicking "dipper down" on the Langmuir control software.

9. Zero the film balance, carefully spreading Solution B drop by drop at the air-water interface (Figure 2A). Make sure lipids are only spreading at the air-water interface, with no chloroform and lipid droplets sinking to the bottom of the polytetrafluoroethylene surface. Failure to ensure this will create a lipid "channel" and prevent monolayer formation.

10. Stop adding lipids till film balance readout around $\sim 15-20$ $\mathrm{mN} / \mathrm{m}$, wait for $\sim 10-15 \mathrm{~min}$. Initiate the barrier controller to alter the surface area by clicking "start experiments," till film balance readout to $37 \mathrm{mN} / \mathrm{m}$. Keep the pressure for 20-30 min (Figure 2B).

11. Raise the cover glass at the speed of $22 \mathrm{~mm} / \mathrm{min}$ while maintaining the surface tension at $37 \mathrm{mN} / \mathrm{m}$. A lipid monolayer with polymer tethering will be transferred from the air-water interface to the surface of cover glass through the Blodgett dipping process (Figure 2C). This forms the bottom leaflet of the lipid bilayer.

12. Clean the air-water interface by suction, rinse the trough with ultrapure water.
13. Clean a one-welled glass slide (e.g., Shaefer slide) using chloroform, ethanol, and ultrapure water before use. Set the clean glass slide on the trough with ultrapure water underneath the water layer. Make sure the well is facing up toward the air-water interface and pour fresh ultrapure water until the glass slide is fully covered. Repeat step 2.8.

14. Hold the cover glass with lipid monolayer from step 2.4 using a silicon suction cup (make sure monolayer side is away from the suction cup), gently push the lipid monolayer to the air-water interface, hold the cover glass for $\sim 2-3 \mathrm{~s}$ at the interface, then push the cover glass against the slide (Figure 2D). Take the slide out with a cover slide.

NOTE: The lipid bilayer will be held at the surface of the cover-glass facing the sandwiched area between the two slides (Figure 2E).

15. Take the cover glass with the bilayer to an epifluorescence microscope. Image the lipid bilayer. If a homogeneous distribution of lipid dye is observed, photobleach a small area of the bilayer for $30 \mathrm{~s}$, turn off the light source for $\sim 30 \mathrm{~s}-1 \mathrm{~min}$., then image again to observe recovery. Lipid bilayer will show fluorescence recovery. NOTE: Membranes with defects or bad fluorescence recovery should not be used for further experiments. 


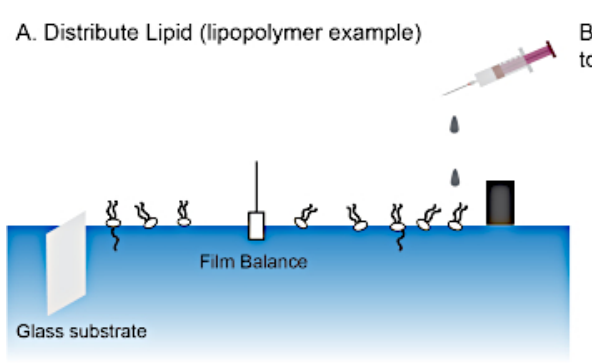

C. Langmuir-Blodgett Dipping

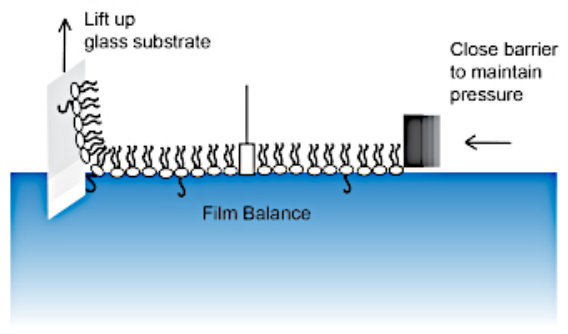

E. Bilayer is sandwiched between cover glass and Schaefer slide
B. Close barrier (transition from a liquid-expanded phase to a liquid condensed phase)

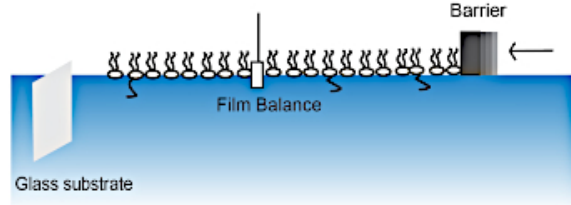

D. Langmuir-Schaefer Transfer

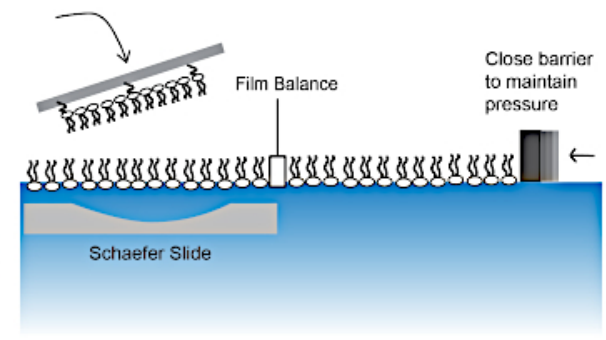

\& Lipopolymer

8 Phospholipid

Figure 2: Steps in making a polymer-tethered lipid bilayer.

Steps of making lipid bilayers using Langmuir-Blodgett dipping (A-C) and Langmuir-Schaefer transfer (D) techniques. (E)

The final "sandwich" containing the lipid bilayer. Please click here to view a larger version of this figure.

\section{Protein reconstitution into the polymer-tethered lipid bilayer}

1. Prepare a crystallization dish containing ultrapure water. Prepare a clean microscope image ring and place underneath the dish.

2. Immerse the "sandwich" of the Schaefer slide and cover glass that containing lipid bilayer underneath the water, gently separate the Schaefer slide and cover glass, hold the cover glass slide from the bottom, away from the bilayer side, transfer the cover glass into the image ring, close the image ring.

NOTE: Make sure that the cover glass with lipid bilayer is always in water, and the ring is well sealed.

3. Replace the ultrapure water in the image ring with Bis-Tris $\mathrm{NaCl}$ buffer, make sure the lipid bilayer is not exposed to any air bubbles. Add $1.1 \times 10^{-9} \mathrm{M}$ n-Octyl- $\beta-\mathrm{D}-$ Glucopyranoside to the lipid bilayer. Immediately add the mixture of $1.2 \times 10^{-9} \mathrm{M}$ of DDM and $1.3 \times 10^{-12} \mathrm{~mol}$ purified I-Opa $1^{36}$ into the image ring. Incubate sample on a benchtop shaker at low speed for $2 \mathrm{~h}$ (Figure 3). 
NOTE: Detergents may vary depending on protein to be reconstituted.

4. Distribute $30 \mathrm{mg}$ SM-2 Resin beads into $3 \mathrm{~mL}$ of Bis-Tris buffer and shake before applying. Use a plastic pipette to add $5 \sim 10 \mu \mathrm{L}$ of SM-2 Resin beads to image ring, incubate for $10 \mathrm{~min}$, remove resin beads by rinsing. The final volume of the buffer in the image ring is $1.5 \mathrm{~mL}$.

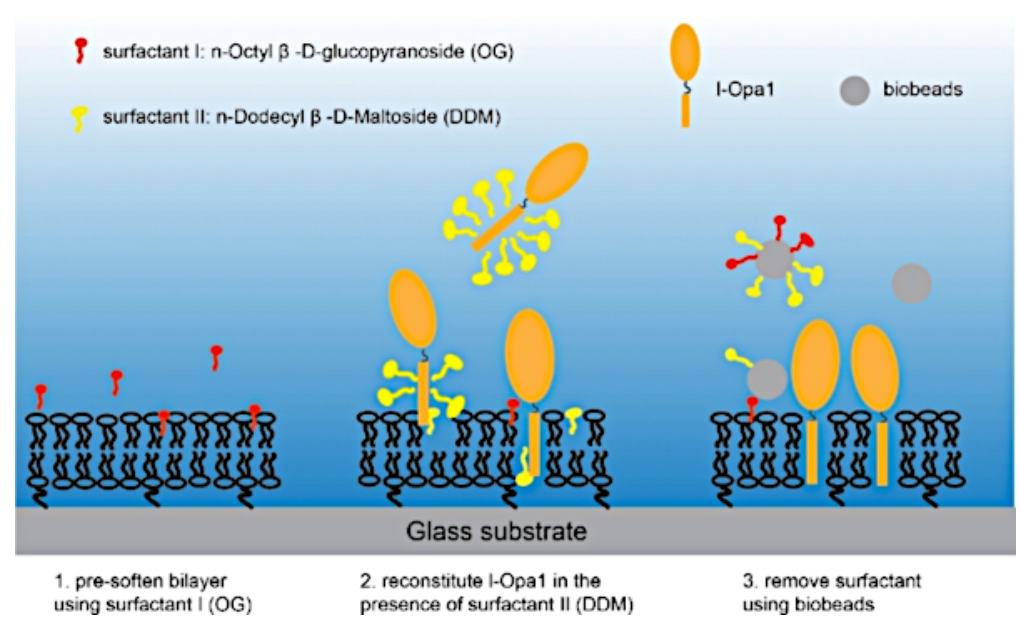

Figure 3: Procedure for reconstituting I-Opa1 into a polymer-tethered lipid bilayer. Please click here to view a larger version of this figure.

\section{Preparation of proteoliposomes}

1. Prepare $1 \mathrm{mg}$ of lipid mixture $\mathrm{A}$ in chloroform solution. Evaporate chloroform under nitrogen flow for $20 \mathrm{~min}$ and keep under vacuum overnight and form a lipid film.

2. Prepare $50 \mathrm{mM}$ calcein containing buffer by dissolving $15.56 \mathrm{~g}$ of calcein to $50 \mathrm{~mL}$ of $1.5 \mathrm{~mol} \mathrm{NaOH}$ solution, stir at room temperature until calcein is completely dissolved, added $12.5 \mathrm{mM}$ Bis-Tris and ultrapure water to the final volume of $500 \mathrm{~mL}$. Adjust the $\mathrm{pH}$ to 7.5 .

3. Suspend lipid film in calcein containing buffer, fully hydrate the lipid by heating the suspension at $65^{\circ} \mathrm{C}$ for $20 \mathrm{~min} .200 \mathrm{~nm}$ liposomes are formed through extrusion using a polycarbonate membrane.
4. Add $2 \mu \mathrm{g}$ of I-Opa1 in $0.5 \mu \mathrm{M}$ DDM to $0.2 \mathrm{mg}$ liposome and incubate at $4{ }^{\circ} \mathrm{C}$ for $1.5 \mathrm{~h}$. Remove the surfactant by dialysis using a $3.5 \mathrm{kDa}$ dialysis cassette against $250 \mathrm{ml}$ of $25 \mathrm{mM}$ Bis-Tris, $150 \mathrm{mM} \mathrm{NaCl}$ and $50 \mathrm{mM}$ calcein buffer at $4{ }^{\circ} \mathrm{C}$ overnight, changing the buffer twice.

5. Remove extra calcein using a PD-10 desalting column.

\section{Imaging and data analysis}

1. Acquire TIRF images using a 100x oil-immersion objective (N.A 1.4). Use $543 \mathrm{~nm}$ laser and a $488 \mathrm{~nm}$ laser for the analysis of TexasRed-PE labeled liposomes and proteoliposomes encapsulated with calcein. Use a 633 $\mathrm{nm}$ laser for the analysis of Cy5-PE embedded in the planar lipid bilayer. 
2. Align the TIRF angle using a lipid bilayer to obtain maximum emission. The quality of lipid bilayer after reconstitution is observed using a 100x oil objective at $25{ }^{\circ} \mathrm{C}$. The diffusion coefficient of phospholipid and reconstituted bilayer are determined using FCS with a protocol describe elsewhere ${ }^{37}$.

3. Add $10 \mu \mathrm{L}$ of $2 \mathrm{mg} / \mathrm{mL}$ proteoliposomes to the image ring and set for 10 mins before the image. GTP, GMPPCP, or GDP are added into the reaction ring with $1 \mathrm{mM} \mathrm{MgCl} 2$ and $1 \mathrm{mM}$ of nucleotide.

4. To determine the influence of s-Opa1 in membrane fusion, titrate s-Opa1 into a proteoliposome/supported bilayer sample containing I-Opa1, and record fusion events.

5. Simultaneous imaging of TexasRed-DHPE and calcein is achieved through a beam-splitting system. Both 488 $\mathrm{nm}$ and $543 \mathrm{~nm}$ lasers are simultaneously applied to the sample as flourescent excitation sources. The emission light is then divided using a $560 \mathrm{~nm}$ beam splitter. The split emission light then is filtered by a $510 \mathrm{~nm}$ filter with a bandwidth of $42 \mathrm{~nm}$ and a $609 \mathrm{~nm}$ filter with a bandwidth of $40 \mathrm{~nm}$. The filtered beam is projected to two adjacent areas on camera chip.

6. Fluorescent emission is simultaneously recorded through a 609-emission filter with a bandwidth of $40 \mathrm{~nm}$, and a 698-emission filter with a bandwidth of $70 \mathrm{~nm}$. The microscope system is equipped with a CMOS camera maintained at $-10^{\circ} \mathrm{C}$.

7. Particle identification of the liposomes can be performed using a Gaussian-based particle recognition algorithm. The particle distribution and intensity are analyzed channel-by-channel. A lipid bilayer signal is used as a mask to isolate particles.

\section{Representative Results}

The reconstituted transmembrane protein freely diffuses and is homogeneously distributed in the membrane.

Example images of a lipid bilayer and its lipid fluidity validated by epifluorescence microscopy is shown in Figure 4. Lipid distribution in bilayer before and after photobleaching is shown in Figure 4A,B. Homogeneity of the lipid bilayer was visualized using an epifluorescence microscope before and after reconstitution (Figure 4D,E). I-Opa1 reconstituted in lipid bilayer was validated by fluorescence correlation spectroscopy (FCS). We use dye conjugated lipids to evaluate the lipid diffusivity of the bilayer. Reconstituted Opa1 was labeled using a fluorescent-tagged anti-Opa1 C-terminal antibody. Bilayer lipid diffusion was measured as $1.46 \pm 0.12$ $\mu \mathrm{m}^{2} / \mathrm{s}$, while the diffusion coefficient of bilayer-reconstituted I-Opa1 was $0.88 \pm 0.10 \mu \mathrm{m}^{2} / \mathrm{s}$. Intensity readout from the FCS curves indicated $75 \%$ of I-Opa1 is reconstituted into the lipid bilayer (Figure 4G,H). These results suggest that I-Opa1 freely diffuses in the polymer-tethered lipid bilayer with the potential to self-assemble into functional complexes. 

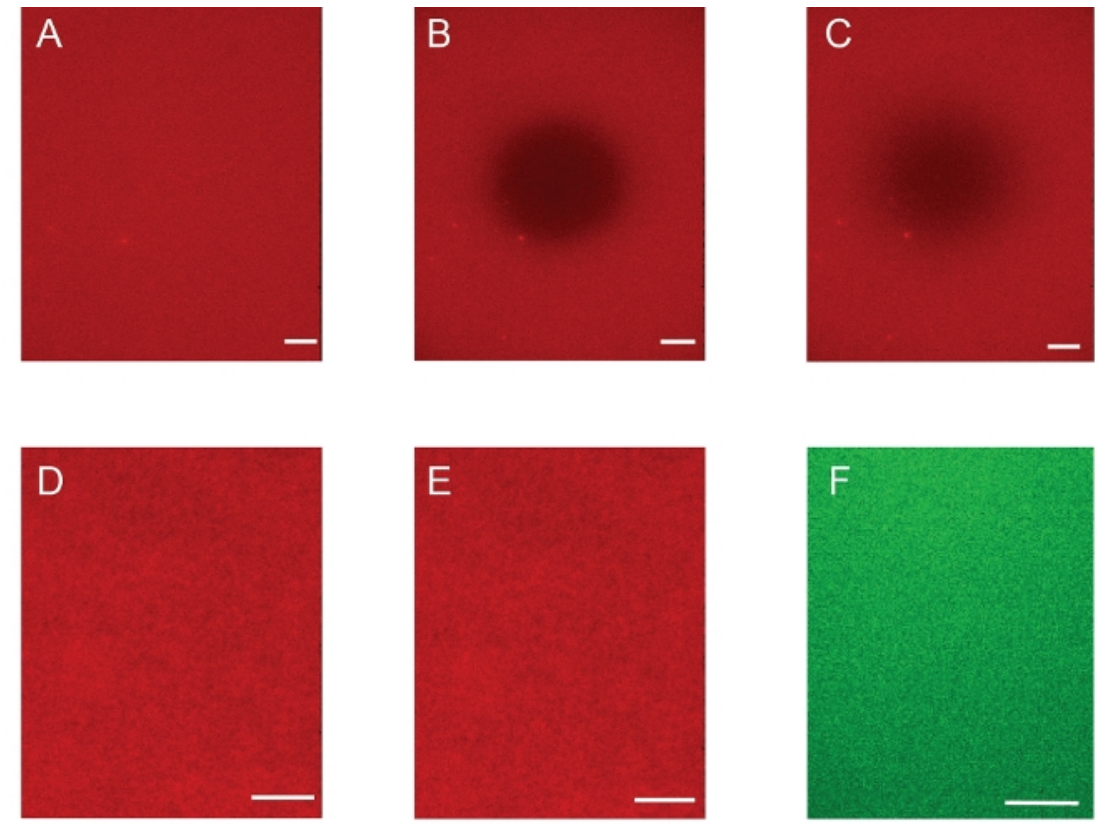

G

$\mathrm{H}$
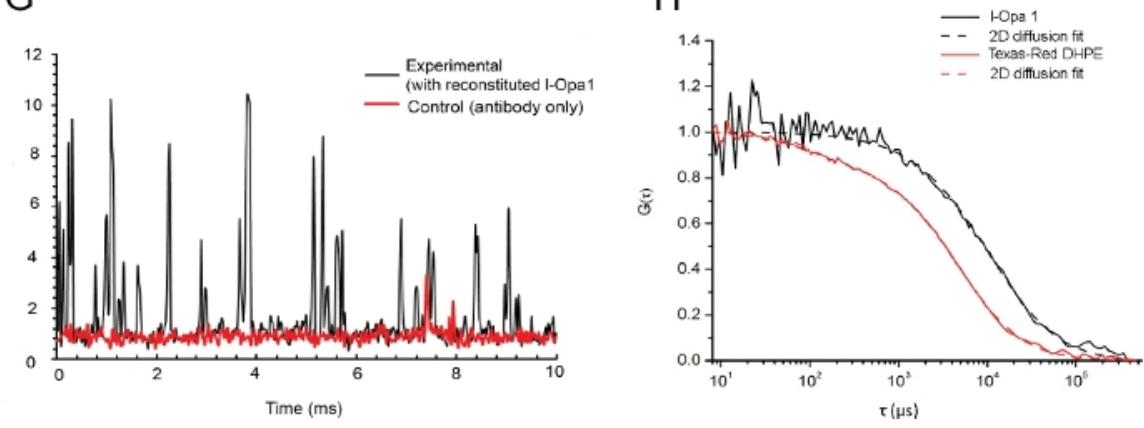

\section{Figure 4: Distribution of lipid and reconstituted protein in the model membrane.}

(A-C) Example images of a lipid bilayer and its lipid fluidity validated by epifluorescence microscopy. (A) Homogeneous lipid distribution in bilayer prior to photobleaching. (B) Snapshot immediately after photobleaching. (C) Bilayer imaged after fluorescence recovery indicates good lipid fluidity of the membrane following reconstitution. (D,E) Representative images of lipid distribution before (D), and after (E) I-Opa1 reconstitution indicate the reconstitution process did not create defects in the bilayer. Representative TIRF image of I-Opa1 labeled with Alexa 488 conjugated antibody (F) showing a homogeneous distribution of Opa1 upon reconstitution. G. Representative raw photon counts of I-Opa1 signal by fluorescent correlation spectroscopy. In the control, no I-Opa1 was reconstituted in the bilayer, while antibody was added and rinsed. The diffusion of I-Opa1 is significantly slower than lipids in the membrane, consistent with successful reconstitution of transmembrane IOpa1 (H). Scale bar: $10 \mu \mathrm{m}$. Please click here to view a larger version of this figure.

Fluorescence step bleaching indicated an average of 2-3 copies of I-Opa1 were reconstituted in a given liposome
(Figure 5A,B). The size distribution of Opa1 reconstituted proteoliposomes was tested after reconstitution using DLS 
(Figure 5C). The reconstitution of Opa1 in proteoliposomes was also verified using FCS. The diffusion coefficient of free antibody was $164 \pm 22 \mu \mathrm{m}^{2} / \mathrm{s}$; diffusion coefficient for liposomes labeled with a lipid dye was $2.22 \pm 0.33 \mu \mathrm{m}^{2} / \mathrm{s}$, and the diffusion coefficient for I-Opa1 proteoliposomes bound to a TexasRed labeled anti-His antibody was $2.12 \pm 0.36 \mu \mathrm{m}^{2} / \mathrm{s}$.

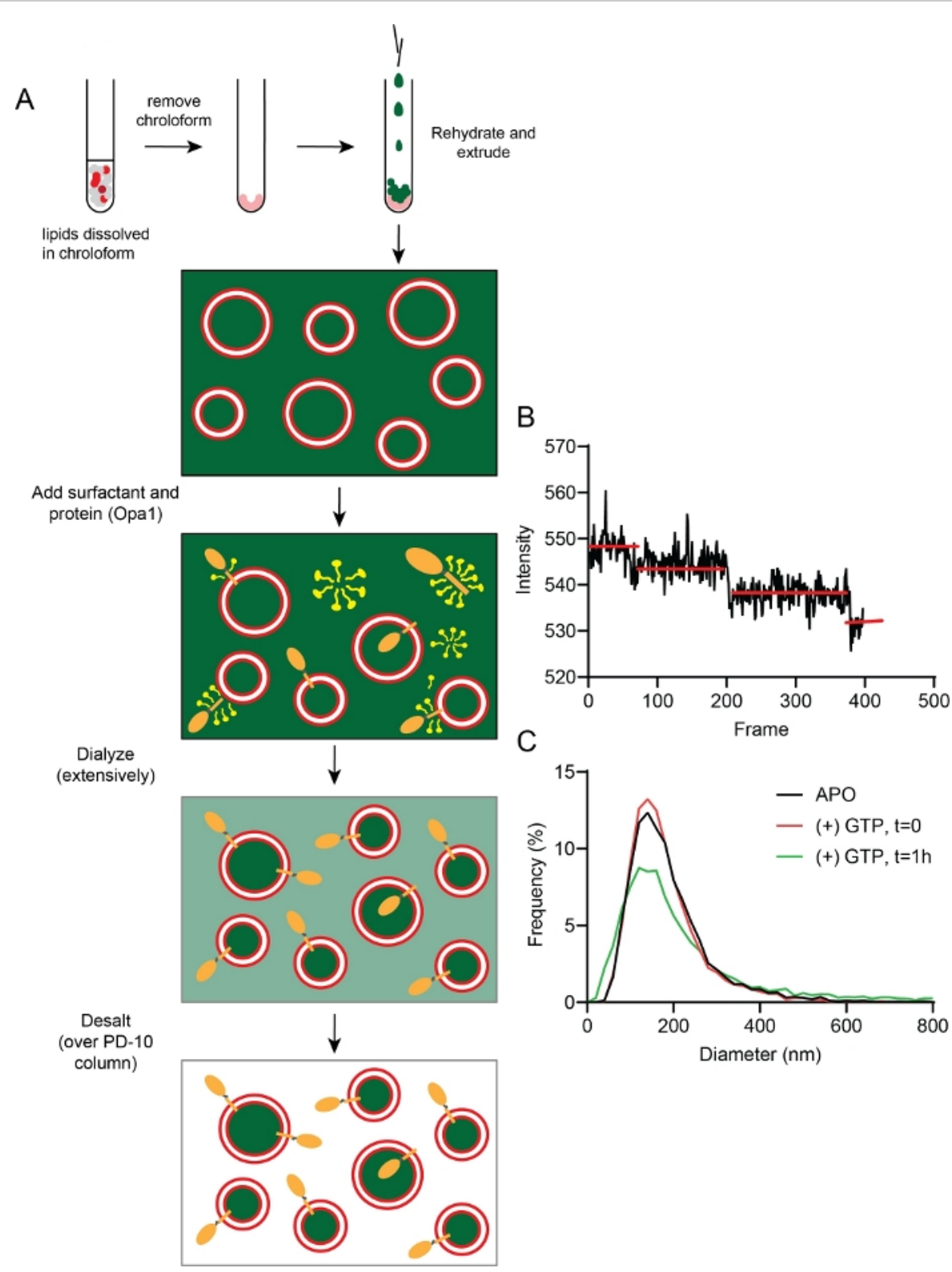

Figure 5: Fabrication and characterization of proteoliposomes.

(A) Steps in fabricating proteoliposomes with encapsulated, quenched calcein. (B) Representative data of fluorescent stepbleaching show an average of 2-3 copies of I-Opa1 embedded in the liposome. (C) Representative size distributions of proteoliposomes (red) without any nucleotide $1 \mathrm{~h}$ after GTP incubation (green). Please click here to view a larger version of this figure. 
Detection of membrane tethering, lipid demixing/ hemifusion, and pore opening by fluorescent microscopy.

Membrane tethering is monitored by observing the signal of TexasRed on the surface of lipid bilayer using TIRF microscopy (Figure 6A). Membrane lipid demixing (hemifusion) behavior was monitored through TexasRed as the dye diffuses from the liposomes into the lipid bilayer. Calcein dequenching helps distinguish full fusion pore formation from only lipid demixing. This allows comparison between conditions where particles stall at hemifusion (Fig $6 \mathrm{~B}$ ), and particles that proceed to full fusion (Figure 6C).
Membrane tethering is indicated by a stable lipid signal from liposomes. The distance could be evaluated based on the FRET signal between the labels of the two membranes ${ }^{36}$. Hemifusion signal features no dequenching in the calcein signal (Figure 6B, lower row), but a rapid decay of the TexasRed signal indicates diffusion of the dye into the lipid bilayer (Figure 6B upper row). Full fusion (with pore opening) features both lipid decay and content release (Figure 6C). TexasRed intensity and calcein intensity can be tracked in a time-dependent manner to provide quantitative detail for the kinetics of membrane fusion ${ }^{36}$. 
A

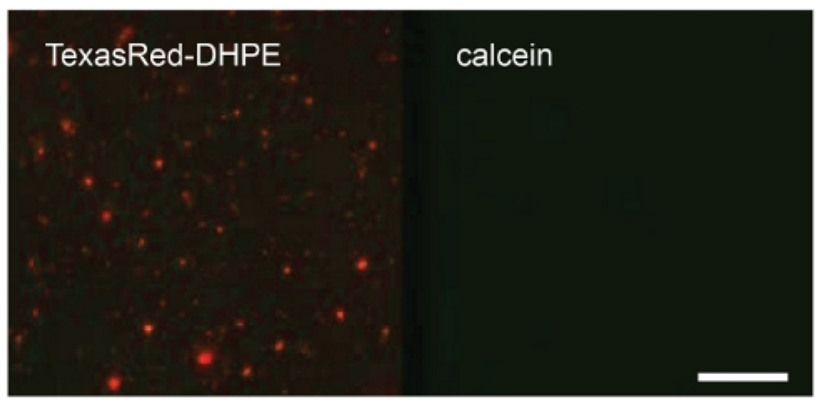

B
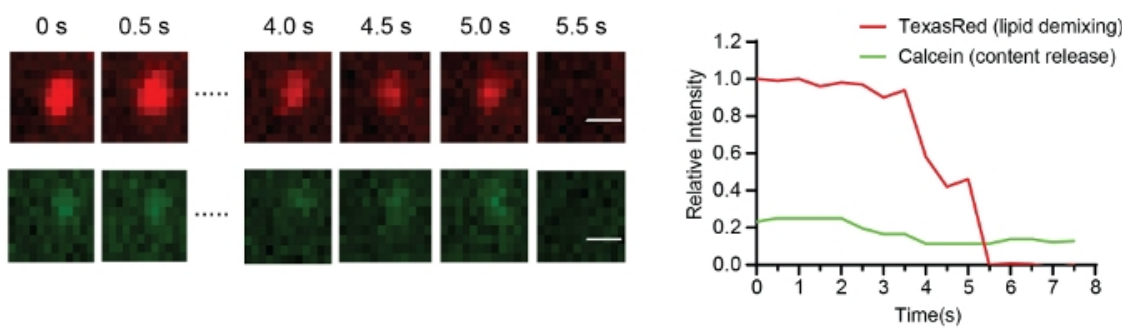

C
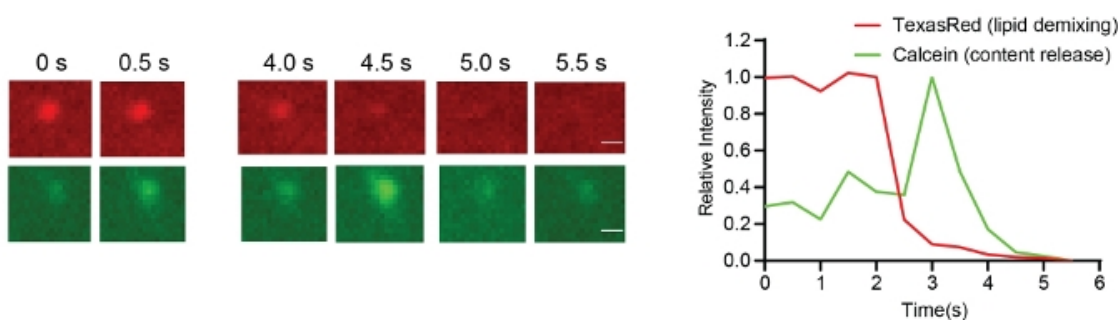

Figure 6: Representative results showing particle tethering (A, scale bar $10 \mu \mathrm{m})$, hemifusion (B, scale bar $0.5 \mu \mathrm{m})$, and fusion (C, scale bar $0.5 \mu \mathrm{m})$.

(A) Proteoliposomes tethered to Opa1-reconstituted lipid bilayer before GTP addition. (B) An example of hemifusion. The upper row in B shows lipid demixing (TexasRed signal, red), lower row in B shows no content release (calcein signal, green) under these conditions. (C) A representative trace of proteoliposome fusing with the lipid bilayer. Content release can be observed from images in the lower row of showing dequenching of calcein (lower row, green). Please click here to view a larger version of this figure.

\section{Discussion}

In vitro model-membrane systems can describe complex membrane processes under well-defined conditions. These systems can distinguish minimal components necessary for complex molecular processes to reveal molecular mechanisms $^{6}, 15,20,38$. For membrane proteins, liposomes and planar supported bilayers are common reconstitution systems. In contrast to solid-supported lipid bilayers, the polymer cushion between the substrate and supported membrane in polymer-tethered bilayers allows free mobility of large membrane proteins, and transmembrane-proteins to diffuse and assemble freely ${ }^{34}$. These features helped us investigate the kinetics of mitochondria inner-membrane fusion ${ }^{36}$ 
We prepared a polymer-tethered lipid bilayer using LangmuirBlodgett/Langmuir-Schaefer (LB/LS) techniques. This allows us to prepare a bilayer with asymmetric lipid components. Cellular membranes have asymmetric leaflet composition, and the LB/LS approach allows the study of such bilayers. With Schaefer transfer, an entire glass substrate can be covered by a lipid bilayer. It is critical to prepare a clean surface for bilayer preparation. Additionally, it takes practice to perform a Schaefer transfer correctly. Unsuccessful Schaefer transfer can create unwanted defects in a lipid bilayer. In this protocol, the pressure added to the film balance is applicable for a bilayer containing $20 \%$ cardiolipin. For bilayers with other components, refer to the surface pressure-area isotherm of the key components. An alternative method is the Langmuir-Blodgett/vesicle fusion (LB/VF) method, where the bottom lipid monolayer is transferred from the air-water interface of a Langmuir trough onto a clean substrate, then liposomes fuse to the top of the supported lipid monolayer and form the final bilayer ${ }^{39}$. Reconstitution of membrane proteins using the LB/VF method is more straightforward than LB/LS, as reconstitution can be performed through the fusion of proteoliposomes. However, vesicle fusion requires the addition of excess liposomes, which may complicate the study of membrane events dependent on concentration-dependent protein-protein interactions.

The successful reconstitution of transmembrane proteins into both polymer-tethered lipid bilayers and liposomes in a preferred functional orientation is important, yet difficult to enforce. Experimental controls are needed to account for this. For polymer-tethered lipid bilayers, it is also important to maintain the integrity of lipid bilayer during reconstitution. Surfactant concentrations must be kept relatively low to prevent dissolving the lipid bilayer, but high enough to prevent denaturation of the protein of interest ${ }^{37,40}$. The method described here is ideal for reconstituting membrane proteins for single-molecule studies but is not necessarily scalable for larger-scale studies. Surfactant choice is another important consideration. Frequently, the surfactant used for purification and storage is a good starting point. The maximum concentration of surfactant is usually $\sim 200$ times less of the $\mathrm{CMC}^{36}$, in a range where the surfactant maintains protein stability and prevents protein aggregation, while maintaining the integrity of membrane ${ }^{36}$. Cocktails containing 2 or 3 surfactants may be considered. For reconstitution into liposomes, a low concentration of surfactant is not necessary. However, surfactant concentrations below CMC are preferable to maintain uniform size and morphology distribution for the liposomes. To prevent leakage of content dye, it is necessary to dialyze against a dye-containing buffer.

In contrast to liposome-based fusion assays, the platform we established provides an approach to investigate the kinetics of each step of membrane fusion. This method provides the ability to study transmembrane fusion proteins under nearnative conditions. Model membrane platforms can be applied to study membrane protein assembly and oligomerization, membrane "sculpting", and protein-lipid interactions of proteins in subcellular environments, like the mitochondrial inner-membrane. This method also allows exploration of important physiological conditions in the membrane-protein interplay, such as bilayer composition asymmetry. The role of a key mitochondrial lipid, cardiolipin, in the bilayer properties of both liposomes and polymer-supported bilayers remains to be defined. Properties such as the ionic strength, membrane thickness, membrane stiffness, membrane curvature, and membrane elastic-viscosity properties all may influence the ability of proteins to assembly into specific functional states. Future studies creatively applying model membrane systems 
have potential to uncover new aspects of membrane protein organization and function.

\section{Disclosures}

None.

\section{Acknowledgments}

The authors acknowledge the support from Charles H. Hood Foundation Child Health Research Award and generous support from the Department of Molecular Biology at Massachusetts General Hospital.

\section{References}

1. Sackmann, E., Biological membranes architecture and function. In Structure and Dynamics of Membranes. Ed: Lipowsky, R., Sackmann, E. Elsiever. 1, 1-63 (1995).

2. Rajendran, L., Simons, K., Lipid rafts and membrane dynamics. Journal of Cell Science. 118 (Pt 6), 1099-1102 (2005).

3. Schink, K. O., Tan, K. W., Stenmark, H. Phosphoinositides in Control of Membrane Dynamics. Annual Review of Cell and Developmental Biology. 32, 143-171 (2016).

4. Gu, R. X., Ingolfsson, H. I., de Vries, A. H., Marrink, S. J., Tieleman, D. P. Ganglioside-Lipid and GangliosideProtein Interactions Revealed by Coarse-Grained and Atomistic Molecular Dynamics Simulations. Journal of Physical Chemistry B. 121 (15), 3262-3275 (2017).

5. Schafer, D. A. Coupling actin dynamics and membrane dynamics during endocytosis. Current Opinion in Cell Biology. 14 (1), 76-81 (2002).
6. Chan, Y. H., Boxer, S. G. Model membrane systems and their applications. Current Opinion in Chemical Biology. 11 (6), 581-587 (2007).

7. Biswas, K. H., Groves, J. T., Hybrid Live Cell-Supported Membrane Interfaces for Signaling Studies. Annual Reviews in Biophysics. 48, 537-562 (2019).

8. Pick, H., Alves, A. C., Vogel, H. Single-Vesicle Assays Using Liposomes and Cell-Derived Vesicles: From Modeling Complex Membrane Processes to Synthetic Biology and Biomedical Applications. Chemical Reviews. 118 (18), 8598-8654 (2018).

9. Kiessling, V., Domanska, M. K., Tamm, L. K., Single SNARE-mediated vesicle fusion observed in vitro by polarized TIRFM. Biophysical Journal. 99 (12), 4047-4055 (2010).

10. Kiessling, V. et al. Rapid fusion of synaptic vesicles with reconstituted target SNARE membranes. Biophysical Journal. 104 (9), 1950-1958 (2013).

11. Yang, S. T., Kiessling, V., Tamm, L. K. Line tension at lipid phase boundaries as driving force for HIV fusion peptide-mediated fusion. Nature Communication. 7, 11401 (2016).

12. Rawle, R. J. van Lengerich, B., Chung, M., Bendix, P. M., Boxer, S. G., Vesicle fusion observed by content transfer across a tethered lipid bilayer. Biophysical Journal. 101 (8), L37-139 (2011).

13. Chao, L. H., Klein, D. E., Schmidt, A. G., Pena, J. M., Harrison, S. C. Sequential conformational rearrangements in flavivirus membrane fusion. Elife. 3, e04389 (2014). 
14. Floyd, D. L., Harrison, S. C., van Oijen, A. M., Analysis of kinetic intermediates in single-particle dwell-time distributions. Biophysical Journal. 99 (2), 360-366 (2010).

15. Sezgin, E.; Schwille, P. Model membrane platforms to study protein-membrane interactions. Molecular Membrane Biology. 29 (5), 144-154 (2012).

16. Ge, Y., Gao, J., Jordan, R., Naumann, C. A. Changes in Cholesterol Level Alter Integrin Sequestration in RaftMimicking Lipid Mixtures. Biophysical Journal. 114 (1), 158-167 (2018).

17. Dutta, D., Kam, L. C. Micropatterned, multicomponent supported lipid bilayers for cellular systems. Methods in Cell Biology. 120, 53-67 (2014).

18. Groves, J., Supported Lipid Bilayers as Mimics for Cell Surfaces and as Tools in Biotechnology. Eds: Ferrari M., Desai T., Bhatia S. BioMEMS and Biomedical Nanotechnology. Springer, Boston, MA (2006).

19. Shoaib, T., Nalam, P. C., He, Y., Chen, Y., EspinosaMarzal, R. M., Assembly, Morphology, Diffusivity, and Indentation of Hydrogel-Supported Lipid Bilayers. Langmuir. 33 (28), 7105-7117 (2017).

20. Wang, N., Rapoport, T. A. Reconstituting the reticular ER network - mechanistic implications and open questions. Journal of Cell Science. 132 (4), jcs227611 (2019).

21. Powers, R. E., Wang, S., Liu, T. Y., Rapoport, T. A. Reconstitution of the tubular endoplasmic reticulum network with purified components. Nature. 543 (7644), 257-260 (2017).

22. Chan, D. C., Fusion and fission: interlinked processes critical for mitochondrial health. Annual Reviews of Genetics. 46, 265-287 (2012).
23. Nunnari, J., Suomalainen, A., Mitochondria: in sickness and in health. Cell. 148 (6), 1145-1159 (2012).

24. Wai, T., Langer, T. Mitochondrial Dynamics and Metabolic Regulation. Trends in Endocrinology Metabolism. 27 (2), 105-117 (2016).

25. Westermann, B. Mitochondrial fusion and fission in cell life and death. Nature Reviews Molecular Cell Biology. 11 (12), 872-884 (2010).

26. Chen, H. et al. Mitofusins Mfn1 and Mfn2 coordinately regulate mitochondrial fusion and are essential for embryonic development. Journal of Cell Biology. 160 (2), 189-200 (2003).

27. Ishihara, N., Fujita, Y., Oka, T., Mihara, K. Regulation of mitochondrial morphology through proteolytic cleavage of OPA1. EMBO Journal. 25 (13), 2966-2977 (2006).

28. Anand, R. et al. The i-AAA protease YME1L and OMA1 cleave OPA1 to balance mitochondrial fusion and fission. Journal of Cell Biology. 204 (6), 919-929 (2014).

29. Mishra, P., Carelli, V., Manfredi, G., Chan, D. C. Proteolytic cleavage of Opa1 stimulates mitochondrial inner membrane fusion and couples fusion to oxidative phosphorylation. Cell Metabolism. 19 (4), 630-641 (2014).

30. Baker, M. J. et al. Stress-induced OMA1 activation and autocatalytic turnover regulate OPA1-dependent mitochondrial dynamics. EMBO Journal. 33 (6), 578-593 (2014)

31. DeVay, R. M. et al. Coassembly of Mgm1 isoforms requires cardiolipin and mediates mitochondrial inner membrane fusion. Journal of Cell Biology. 186 (6), 793-803 (2009).

32. Rainbolt, T. K., Lebeau, J., Puchades, C., Wiseman, R. L. Reciprocal Degradation of YME1L and OMA1 
Adapts Mitochondrial Proteolytic Activity during Stress.

Cell Reports. 14 (9), 2041-2049 (2016).

33. Ban, T. et al. Molecular basis of selective mitochondrial fusion by heterotypic action between OPA1 and cardiolipin. Nature Cell Biology. 19 (7), 856-863 (2017).

34. Tanaka, M., Sackmann, E., Polymer-supported membranes as models of the cell surface. Nature. 437 (7059), 656-663 (2005).

35. Shilts, K., Naumann, C. A., Tunable cell-surface mimetics as engineered cell substrates. Biochimica Biophysica Acta Biomembrane. 1860 (10), 2076-2093 (2018).

36. Ge, Y. et al. Two forms of Opa1 cooperate to complete fusion of the mitochondrial inner-membrane. Elife. 9, e50973 (2020).

37. Ge, Y., Siegel, A. P., Jordan, R., Naumann, C. A. Ligand binding alters dimerization and sequestering of urokinase receptors in raft-mimicking lipid mixtures. Biophysical Journal. 107 (9), 2101-2111 (2014).

38. Simons, K., Vaz, W. L. Model systems, lipid rafts, and cell membranes. Annual Review of Biophysics and Biomolecular Structure. 33, 269-295 (2004).

39. Kiessling, V., Liang, B.; Tamm, L. K. Reconstituting SNARE-mediated membrane fusion at the single liposome level. Methods in Cell Biology. 128, 339-363 (2015).

40. Siegel, A. P., Kimble-Hill, A., Garg, S., Jordan, R., Naumann, C. A. Native ligands change integrin sequestering but not oligomerization in raft-mimicking lipid mixtures. Biophysics Journal. 101 (7), 1642-1650 (2011). 